\title{
TP53 and NRAS are the Most Frequently Mutated Genes in Stool DNA of CRC Patients from Central Part of Iran
}

\author{
Farideh Saberi \\ Isfahan University of Medical Sciences \\ Virinder Sarhadi \\ University of Helsinki \\ Omar Youssef \\ University of Helsinki \\ Arto Kokkola \\ University Central Hospital of Helsinki \\ Pauli Puolakkainen \\ University Central Hospital of Helsinki \\ Sakari Knuutila \\ University of Helsinki \\ Rasoul Salehi ( $\square$ r_salehi@med.mui.ac.ir) \\ Isfahan University of Medical Sciences
}

\section{Research Article}

Keywords: Stool DNA, colorectal cancer, mutations, next generation sequencing

Posted Date: September 17th, 2021

DOI: https://doi.org/10.21203/rs.3.rs-792966/v1

License: (a) (1) This work is licensed under a Creative Commons Attribution 4.0 International License. Read Full License 


\section{Abstract}

Colorectal cancer (CRC) rated among the three most diagnosed cancers and the fourth main cause of death worldwide. CRC is a curable cancer provided to be diagnosed at its early-stage. Colonoscopy, stool and blood-based tests are in use for CRC diagnosis/screening. Due to low patient compliance, low specificity and high rate of false results, more reliable methods with desired level of detection accuracy and high patients' compliance are highly demanded. Detecting hotspot mutations in stool DNA emerged as a robust noninvasive alternative, but due to the different genetic background of various populations and hence varied mutation spectrum/prevalence, prior assessment of CRC mutations in the population is essential. Here, we have evaluated stool DNAs from CRC patients and controls using a NGS based 22 genes panel. Hotspot mutations in NRAS, FGFR3, SMAD4 and TP53 genes had higher prevalence among the CRC patients compare to normal controls. Patients were followed up in their post-surgical period. Six of them (12\%) with TP53 mutations (2 patients had NRAS mutation as well) were died of cancer. Those harboring mutations in TP53 and/or NRAS would be regarded as high risk and should be provided with special care.

\section{Introduction}

Colorectal cancer (CRC) is one of the common malignancies worldwide. Incidence and mortality rates are still high and the number of deaths due to CRC are expected to increase to 1.1 million by $2030^{1}$.

Usually precursors of CRC are benign adenomatous colonic polyps (adenomas) ${ }^{2}$. Burden of adenomas are depending on factors like their size, location and malignant grade. Transformation from adenoma to CRC is a decade long stepwise and gradual process ${ }^{3}$ with its early detection and resection considerably reduces CRC incidence and mortality 4,5 . The gradual nature and accumulation of different genetic alterations during a comparatively long period of time creates excellent opportunity for screening through colonoscopic surveillance. Therefore, $\mathrm{CRC}$ is well compatible with the World Health Organization criteria for a diseases best fitted for screening ${ }^{6}$. If high risk patients for CRC undergo regular surveillance colonoscopy and affected individuals have access to improved surgical procedures and adjuvant therapies, CRC mortality would reduce considerably ${ }^{7}$.

The five-year CRC survival rate is as high as $90 \%$ for earlier stages localized cancer but decrease to even less than $5 \%$ while approaching to late stage metastatic disease ${ }^{8}$. In spite of CRC screening proved beneficial for early detection of CRC ${ }^{8,9}$ and resulted in reduction of CRC mortality and incidence, but still participants in screening programs remains too low; $65 \%$ in the US ${ }^{10}$ and from $1.9-54 \%$ across Europe OECD: Screening, survival and mortality for colorectal cancer ${ }^{11}$. The situation is even more disappointing when considering CRC screening rates in underdeveloped countries. Analysis of 10 years data from Germany regarding individuals' attitude to undertake colonoscopy for CRC screening was $23 \%$ for men and $26 \%$ for women ${ }^{12}$. Further analysis of the German patients revealed that in addition to colonoscopy, inclusion of noninvasive tests in screening and availability of broader range of screening choices, compliance and interest to undertaking CRC screening increased from around $25 \%$ to as high as $98 \%{ }^{13}$.

Gradual accumulation of driver mutations over the time will ultimately manifested as a recognizable tumor. Therefor molecular alterations in the colonic epithelium remained undetectable by colonoscopy unless the accumulated genome mutations represent as a distinct morphological abnormality which is late and shows limitation of colonoscopy ${ }^{14}$. Therefore, normal colonoscopy outcome for an individual doesn't exclude molecular alterations in the colon epithelium. Finding driver mutations in stool sample of CRC patients or those diagnosed as normal individuals in colonoscopy examination would be helpful to further recognize those at higher CRC risk based on their mutation status ${ }^{14}$.

It is demonstrated that stool DNA assay is a simple, non-invasive method for analyzing genetic alterations in patients with gastrointestinal neoplasms ${ }^{15}$. Tumors shed more cells in the large-bowel lumen than normal epithelium lining and shedding occurs at all stages of tumor initiation and progression, making it an appropriate target for analyzing pathological in the DNA and can be a reliable approach for early detection of malignancies ${ }^{16,17}$. A multi target stool DNA assay for CRC screening was approved by the FDA in 2014 and its application has also increased rapidly in other countries variations ${ }^{18-20}$.

Variability exists between different populations regarding underlying molecular etiology of CRC. Comparison of Middle East and Western populations revealed the aspects of inconsistency in CRC pathways and mutated genes ${ }^{21}$. Iran, like many other countries especially in Asian continent, is inhabited by a multi ethnic genetically heterogeneous population. Comparison of mutation spectrum of beta thalassemia as a most prevalent single gene disorder in Iran, and many other countries, confirmed the extreme genetic heterogeneity of Iranian population ${ }^{22}$. Therefore, need to study extensively and comparative studies regarding molecular drivers of CRC between Iranian 
patients and those with different genetics background, like Western Europeans would be beneficial. For this reason we compared mutation spectrum of Iranian CRC patients and controls with Finnish CRC patients as a standard European population performed in the same laboratory at Helsinki university ${ }^{23}$.

In the present study, we performed mutation analysis using a 22 gene targeted NGS panel on DNA extracted from stool samples of early stages of CRC patients and their healthy counterparts from central region of Iran, then the patients and control group were followed up. The outcome of this study may facilitate the development of stool-based modality for population specific early-stage CRC risk prediction. Beside colonoscopy which is a gold standard diagnostic/surveillance method, availability of other noninvasive with desired level of detection sensitivity for screening approaches would encourage at risk individuals to undertake regular screening tests.

\section{Results}

The success rate of amplicon based NGS analysis from stool samples was $96 \%$ and $98 \%$ for patients and controls respectively. Two CRC samples and one normal sample were removed from NGS assay because of poor DNA quality. Among the CRC patients, 18 patients diagnosed with stage I and rest of them categorized as stage II CRC. Average age of CRC patients and controls was 62.8 years (range $43-$ 87 ) and 64.6 years (range $49-88$ ) respectively. The majority of participants were male (62\%). The location of the tumor was more frequent in colon. The characteristics of all 99 individuals included in the study are presented in Table 1.

Table 1

Characteristics of patients and healthy controls included in the study

\begin{tabular}{|lllll|}
\hline & CRC & Normal & CRC & Normal \\
& Iranian & Iranian & Finnish & Finnish \\
\hline Male & $\mathrm{N}=31$ & $\mathrm{~N}=28$ & $\mathrm{~N}=21$ & $\mathrm{~N}=4$ \\
\hline Female & $\mathrm{N}=19$ & $\mathrm{~N}=21$ & $\mathrm{~N}=12$ & $\mathrm{~N}=9$ \\
\hline Average age (years) & $62.8($ range 43-87) & 64.6 (range 49-88) & 72 (range 53-85) & 44 (range 19-65) \\
\hline Stage & $\mathrm{I}=18$ & & & \\
\hline Rectal cancer & $\mathrm{N}=32$ & & $\mathrm{~N}=22$ & \\
\hline Colon cancer & $\mathrm{N}=10$ & & $\mathrm{~N}=11$ & $\mathbf{1 3}$ \\
\hline Total & $\mathrm{N}=40$ & & 33 & \\
\hline
\end{tabular}

Among the CRC patients, mutations were seen in 24/50 patients (48\%) and in the 13/49 (26.5\%) control samples. Hotspot mutations were noted in 16/50 patients and 7/49 controls. Four of the patients and two of the control samples had more than three mutations. Rest of the samples detected with no mutation with the NGS gene panel we used and need to be evaluated by whole exome sequencing (WES) in order to pinpoint the underlying molecular defects.

Out of 22 genes in the lon AmpliSeq panel, mutations were seen in 19 genes in patients and 17 genes in control samples; mutations in MAP2K and NRAS were seen only in patients and not in controls. A total of 34 hotspot mutations and 37 novel mutations were detected in patient samples, while 13 hotspots and 34 novel mutations were detected in control individuals (Fig. 1).

TP53 was the most frequently mutated gene in patients with a total of 13 mutations detected, while SMAD4 and DDR2 were the most frequently mutated genes in controls, with 7 mutations in each gene. All mutations in TP53 were hotspot mutations. Among the patients, hotspot mutations were most common in TP53, followed by NRAS, FGFR3 and SMAD4 (Table 2). Most of the mutations in controls were novel and only seven individuals had hotspot mutations. The AKT, CTNNB1 and NOTCH1 genes had no mutation in both groups.

The recurrently occurring hotspot mutations among patients were codon 273 of TP53, seen in 4 patients (2 with R273C, 2 with R273H) and NRAS codon 12 (3 patients) and codon 61 mutations (2 patients).

The entire list of mutations (COSMIC and novel) in patients and normal individuals can be found as Supplementary Table S1 online. 
Table 2

Comparison of hotspot mutations detected in stool DNA of Iranian and Finnish colorectal cancer patients and controls

\begin{tabular}{|c|c|c|c|c|c|c|}
\hline \multirow[b]{2}{*}{ Gene } & \multicolumn{2}{|c|}{ Finnish CRC } & \multicolumn{2}{|c|}{ Iranian CRC } & \multicolumn{2}{|c|}{ Iranian Controls } \\
\hline & Patient & Mutation & Patient & Mutation & Individual & Mutation \\
\hline AKT1 & 28 & E17K & & & & \\
\hline ALK & & & & & C16 & E1197K \\
\hline \multirow[t]{2}{*}{ KRAS } & 55 & G12V & T15 & G13V & $\mathrm{Cg}$ & D119G \\
\hline & 28 & G12D & & & & \\
\hline BRAF & & & T1 & G606R & C16 & T599I \\
\hline \multicolumn{7}{|c|}{ CTNNB1 } \\
\hline \multicolumn{7}{|l|}{ ERBB2 } \\
\hline EGFR & & & & & C43 & Q486* \\
\hline \multirow[t]{2}{*}{ ERBB4 } & & & T1 & E317K & C9 & S239L \\
\hline & & & T9 & C304F & & \\
\hline FBXW7 & & & T45 & H379P & & \\
\hline \multicolumn{7}{|l|}{ NRAS } \\
\hline & & & T13 & G13V & & \\
\hline & & & $\mathrm{T} 4$ & Q61K & & \\
\hline & & & T5 & Q61K & & \\
\hline & & & T40 & G12V & & \\
\hline & & & T9 & G12S & & \\
\hline & & & T43 & G12V & & \\
\hline PIK3CA & & & T9 & A1066T & C7 & A1020V \\
\hline PTEN & & & & & C16 & R234Q \\
\hline \multirow[t]{3}{*}{ SMAD4 } & & & T43 & R361H & & \\
\hline & & & T9 & $\mathrm{R} 135^{\star}$ & & \\
\hline & & & T9 & $\mathrm{R} 420 \mathrm{H}$ & & \\
\hline STK11 & 22 & F354L & & & $\mathrm{C9}$ & A205T \\
\hline FGFR1 & & & T9 & A299V & $\mathrm{C9}$ & D165DD \\
\hline \multicolumn{7}{|l|}{ FGFR2 } \\
\hline \multirow[t]{3}{*}{ FGFR3 } & & & $\mathrm{T} 1$ & V392M & & \\
\hline & & & T27 & S373G & & \\
\hline & & & T23 & F386L & & \\
\hline \multirow[t]{2}{*}{ MET } & & & T9 & S349G & & \\
\hline & & & T23 & M1268T & & \\
\hline \multicolumn{7}{|c|}{ NOTCH1 } \\
\hline \multirow[t]{2}{*}{ TP53 } & 12 & P72A & $\mathrm{T} 1$ & E358K & $\mathrm{C} 23$ & T230A \\
\hline & 31 & Y205D & T1 & G279E & C33 & R273C \\
\hline
\end{tabular}




\begin{tabular}{|c|c|c|c|c|c|}
\hline \multicolumn{2}{|c|}{ Finnish CRC } & \multicolumn{2}{|c|}{ Iranian CRC } & \multicolumn{2}{|c|}{ Iranian Controls } \\
\hline 55 & T172fs & T10 & P151S & C9 & E285K \\
\hline \multirow[t]{10}{*}{68} & Y163H & T27 & Y205H & $\mathrm{C} 10$ & M169T \\
\hline & & T3 & $\mathrm{R} 273 \mathrm{C}$ & & \\
\hline & & T5 & E286K & & \\
\hline & & $\mathrm{T} 4$ & R248W & & \\
\hline & & T6 & $\mathrm{R} 273 \mathrm{H}$ & & \\
\hline & & $\mathrm{T} 40$ & R248W & & \\
\hline & & $\mathrm{T} 27$ & N235S & & \\
\hline & & T52 & $\mathrm{R} 273 \mathrm{C}$ & & \\
\hline & & T44 & G245S & & \\
\hline & & T43 & $\mathrm{R} 273 \mathrm{H}$ & & \\
\hline
\end{tabular}

\section{Mutations in Finnish cohort:}

Among the Finnish CRCs patients, hotspot mutations were seen in 6/33 patients (18\%) and two novel mutations were seen in the control group. All mutations in CRC group were hotspot and two patients had more than one mutation, whereas no hotspot mutation was seen in the control group. Out of the 22 genes in the lon AmpliSeq panel, eight mutations were seen in four genes, AKT1, KRAS, STK11 and TP53 in six patients. TP53 was the most commonly mutated gene followed by KRAS (Table 2).

\section{Comparison of hotspot mutations in Iranian and Finnish CRC patients and controls}

Although data related to Finnish patients was published already but for the sack of comparison of two typical ethnic representatives of Middle-East and Europe, the results of stool DNA analysis of the Finnish cohort which have been worked out by the same gene panel, we review and included their results here. Overall, higher proportion of Iranian patients showed mutations in their stool DNA compared to Finnish patients ( $32 \%$ and $18 \%$ respectively), with 34 mutations detected in $32 \%$ Iranian patients compared to eight mutations in $18 \%$ Finnish patients. TP53 was the most commonly mutated gene in both the Iranian and Finnish cohorts. The second most commonly mutated gene was a RAS gene, being NRAS in Iranian patients and KRAS in Finnish patients. The frequency of NRAS mutations was significantly higher in Iranian patients than that in Finnish patients. Mutations in $A K T 1$ were noted only in Finnish patients while mutations in MET, FGFR3, SMAD4, NRAS, FBXW7, were seen only in Iranian patients (Fig. 2).

Similar to the pattern seen for patients, mutations were more frequent in Iranian control group, with 7 out of 49 healthy Iranian individuals showed mutations, while no hotspot mutations were seen among Finnish healthy individuals.

\section{Iranian patient's treatment}

From the CRC group, 29 patients (18 patients with stage I and 11 patients from 32 patients with stage II of cancer) were in group A and only did surgery according to their pathology reports, while 21 patients in group B received adjuvant treatment in addition to surgery. From group A one patients died of independent cancer (DOIC) with no hotspot mutation and two patients died of cancer (DOC), one patient had hotspot mutation in TP53 and one patient with novel mutations in ERBB2, FGFR3 and MET genes. From group B, 6 patients (12\%) were died. DOC (one patient with COSMIC mutations in NRAS, ERBB4, MET, PIK3CA, FGFR1 and SMAD4 genes and numerous novel mutations in other genes, one patient with COSMIC mutations in BRAF, ERBB4, FGFR3 and TP53 genes in addition to numerous novel mutations, one patient with COSMIC mutations in SMAD4, TP53 and NRAS genes, two patients only with TP53 mutation and one patient with mutations in TP53 and NRAS genes). From this group one patient was DOIC with no mutation (Table 3). 
Table 3

Following up data of the CRC patients and characteristics of those how died due to cancer

\begin{tabular}{|c|c|c|c|c|c|c|c|c|}
\hline & Code & DOIC/DOC* & Stage/TNM & Grade $^{\wedge}$ & Histological type & $\begin{array}{l}\text { P53 } \\
\text { COSMIC } \\
\text { mutation }\end{array}$ & $\begin{array}{l}\text { NRAS } \\
\text { COSMIC } \\
\text { mutation }\end{array}$ & Other COSMIC mutations \\
\hline \multirow{3}{*}{$\begin{array}{l}\text { Group A } \\
\text { (29 } \\
\text { Patients) }\end{array}$} & T10 & DOC & I/T2NOMX & $\mathrm{p}$ & Adenocarcinoma & yes & no & no \\
\hline & T30 & DOC & I/T1NOMX & w & $/ /$ & no & no & no \\
\hline & $\mathrm{T} 2$ & DOIC & I/T1NOMX & w & $/ /$ & no & no & no \\
\hline \multirow{7}{*}{$\begin{array}{l}\text { Group B } \\
\text { (21 } \\
\text { Patients) }\end{array}$} & $\mathrm{T} 1$ & DOC & II/T4NOMX & $\mathrm{p}$ & $/ /$ & yes & no & FGFR3,ERBB4,BRAF \\
\hline & T3 & DOC & II/T3NOMX & $\mathrm{p}$ & $/ /$ & yes & no & no \\
\hline & T9 & DOC & II/T3NOMX & w & // & no & yes & \multirow{2}{*}{$\begin{array}{l}\text { ERBB4,MET,FGFR1,SMAD4, } \\
\text { PIK3CA }\end{array}$} \\
\hline & $\mathrm{T} 40$ & DOC & II/T3NOMX & $\mathrm{p}$ & $/ /$ & yes & yes & \\
\hline & T43 & DOC & II/T3NOMX & $\mathrm{p}$ & $/ /$ & yes & yes & \multirow{2}{*}{$\begin{array}{l}\text { no } \\
\text { SMAD4 }\end{array}$} \\
\hline & T44 & DOC & II/T4aNOMX & $\mathrm{p}$ & $/ /$ & yes & no & \\
\hline & T48 & DOIC & II/T3NOMX & w & $/ /$ & no & no & no \\
\hline
\end{tabular}

Also, the follow up results showed that three normal individuals (more than $6 \%$ ) encountered gastrointestinal complications such as bleeding and abdominal pain after 33 months. All these three individuals were detected with mutations (one patient in BRAF, PTEN and ALK genes, one patient in KRAS, ERBB4, STK11, FGFR1 and TP53 genes and one patient only in TP53 gene). One of the controls detected with mutation in five genes showed polyps $(4 \mathrm{~mm})$ in his recent colonoscopy examination. Also, one of the control samples detected with two novel mutations in FGFR3 gene and three mutations in SMAD4 and other novel mutations, had gastrointestinal complications in follow up.

\section{Discussion}

We compared mutations detected in DNA from stool of CRC patients and healthy individuals. Applying NGS analysis on stool DNA from CRC patients and healthy individuals using the lon AmpliSeq Colon and Lung Cancer panel we were able to successfully get results in $96 \%$ of patients, thus overcoming the problem of low DNA quantity/quality associated with stool analysis 24,25 .

From the DOC patients, who had only TP53 mutations, two patients were DOC and one patient had metastases approximately 24 months after their therapy. Moreover, $\% 18$ of patients had double mutations and two of them had mutation in both NRAS and TP53 genes who were DOC. It can show that mutation in TP53 gene or both TP53 and NRAS genes could reduce patient's survival compare to patients with no mutation or mutation in other genes. A study in 2014 showed that patients with mutation in KRAS, NRAS, TP53 and PIK3CA genes had dramatically shorter survival than patients with any other mutations ${ }^{26}$. These results also showed that patients with more than one mutation had lower survival rate than patients with one mutation or no mutation ${ }^{26}$.

Interestingly NRAS mutations seen in 6 patients (12\%), four females with codon 12 (p.G12S, p.G12V) or 13 (p.G13V) (mean age 66 years) in stage II of primary tumor located in left colon. A female and a male patient detected with codon 61 (p.Q61K) mutations. Cercek et al found that patients with NRAS mutations, particularly in exon 3, comprised aggressive subset of metastatic colorectal cancer with overall reduced survival than patients with KRAS mutations or RAS wild-type tumors. They also showed that African Americans CRC patients with NRAS mutations are mostly located at left side colon ${ }^{27}$. In a large scale study on Iranian metastatic CRC specimens from 1000 patients using pyrosequencing and high resolution melting (HRM) analysis, KRAS mutations detected in $33.6 \%$ cases (codon 12 and 13 ) ${ }^{28}$. Another study from west of Iran analyzed the KRAS, BRAF and NRAS genes on formalin fixed paraffin embedded (FFPE) tissues from 33 CRC patients showed KRAS mutations (codon 12 and 13 ) in 12 patients (27.3\%) and no mutations in NRAS and BRAF ${ }^{29}$. Similarly, another study from Northeastern region of Iran showed that none of the 87 tissue samples from CRC patients had mutation in NRAS gene which is similar to the results from West part of Iran ${ }^{30}$. Data from 7 European countries showed that mutations in NRAS is predictors of lower response to anti-EGFR MoAbs treatment in CRC ${ }^{31}$. Jouini et al showed that NRAS gene mutated in $6.9 \%$ of the 129 Tunisian colorectal cancer specimens ${ }^{32}$. In Finnish stool DNAs, only one patient with rectum adenoma showed NRASQ61R mutation ${ }^{24}$, while in another cohort of stool samples from Iranian CRC patients, none of them showed NRAS mutation in codon 12,13 or $61^{25}$. All the three studies just 
mentioned, performed by the same NGS gene panel in the same laboratory at Helsinki. The exact reason for the higher frequency of NRAS mutations in the present Iranian cohort is not clear. The samples for this study were collected mainly from Isfahan province located at the central part of Iran while samples for earlier Iranian cohort were mainly collected from Tehran region ${ }^{25}$. A study on RAS mutations in 353 Chinese CRC patients showed that NRAS alterations in tumor tissues were more frequent in female CRC patients than in male patients $(75.0 \%$ vs $41.1 \%)$, which is quite similar to the observation in our study, with 5 out of 6 Iranian patients with NRAS mutations were female 33. NRAS mutations reported to be associated with poor prognosis and resistance to anti-EGFR monoclonal antibodies in CRC patients. A retrospective European Consortium analysis of 1022 tumor DNA samples of metastatic CRC found mutations in $40 \%$ of $K R A S$, in $2.6 \%$ of NRAS, in $4.7 \%$ of BRAF and in $14.5 \%$ of PIK3CA. In wild type KRAS patients, the presence of any mutations in NRAS, BRAF or PIK3CA was associated with lower response rates, disease control rates and shorter overall survival. These findings suggested that up to $60-65 \%$ of patients with wild type KRAS tumors are resistant to anti-EGFR agents, which is accounted for presence of other mutations ${ }^{31,34}$. An NGS study on 91 Brazilian CRC patients demonstrated that driver mutation in the APC, TP53 and KRAS genes were significantly high in CRC patients ${ }^{35}$. They also showed that at least one gene involved in the MAPK-ERK pathway (KRAS, BRAF, and NRAS) had mutation in $68 \%$ of cases. Another study on 145 CRC patients by targeted deep sequencing showed that the TP53 gene was most commonly mutated gene and was significantly linked to lower disease-free survival which is similar to our study ${ }^{36}$. However, in TCGA datasets $A P C$ mutation is mostly common followed by TP53 and KRAS mutations.

In our study, detection of relatively high percentage of NRAS mutation (12\%) among CRC samples from central part of Iran would emphasis the necessity of paying more attention to NRAS mutation analysis at least for patients from this particular ethnicity. Our stool DNA mutation analysis showed some aspects of uniqueness which would be helpful for more accurate personalized treatment decision and development of early population specific noninvasive CRC screening tests.

To find out the likelihood of developing CRC in control individuals with normal colonoscopy results but detected with mutations in their stool DNA we organized a following up program. As mentioned earlier, hotspot mutations detected in 7 non-affected control individuals (Table 2). Although based on the colonoscopy examination they categorized as unaffected normal individuals but later in follow up period 3 of them developed polyps and experienced polypectomy. It is obviously showing the importance of genetic analysis for those who are undergoing colonoscopy even though emerging with normal colonoscopy results. Recent studies analyzing the mutations in normal individuals have shown that mutations increased with age in colon tissue ${ }^{37,38}$. Since colonoscopic report for these 7 individuals reflect a polyp free condition, presence of some particular hotspot mutations in their stool DNA analysis emphasis the necessity of surveillance colonoscopy at regular time intervals for timely detection of any probable polyp development.

Some mutations reported to be an early event in tumor development ${ }^{14}$. Presence of tumor driver mutations in normal individuals related to tumor initiation and progression like KRAS, ERBB gene family, TP53 and so, are important criteria based on which we can categorize normal individuals as high-risk group for developing CRC in future and regular colonoscopy surveillance should be recommended.

\section{Materials And Methods}

\section{Patients and stools collection and follow up}

\section{Iranian samples:}

The stool samples collected from 52 patients diagnosed with CRC by colonoscopy before starting any cancer treatment and 50 healthy individuals with normal colonoscopy results. Samples were collected from July 2016 to December 2017 at Alzahra university hospital, Isfahan Iran. The study was approved by Isfahan University of Medical Sciences Ethics Committee and informed written consent was obtained from all participants recruited to this study. Stool samples were stored at $-80^{\circ} \mathrm{C}$ until DNA extraction. Patients followed up since their first surgery. The median follow-up period was 30 months (range 23-39 months), for this aim patients were classified in two groups based on their pathology report (stage I and II). This study was approved by ir.mui.rec.1394.3.936 code at Isfahan University of medical science ethnical committee. All procedures performed in studies involving human participants were in accordance with the ethical standards of the institutional and/or national research committee and with the 1964 Helsinki declaration and its later amendments or comparable ethical standards. Informed consent was obtained from all individual participants included in the study.

\section{Finnish samples:}

The stool samples from Finnish CRC patients were collected between April 2015 to May 2017 from patients referred to Kirugi, Meilahti and Jorvi hospitals in Finland. The sequencing results of stool DNA samples collected from 33 CRC patients and 13 healthy individuals 
(Table 1) of Finnish origin published previously 24 .

\section{Mutation analysis by NGS \\ DNA isolation}

QIAamp DNA stool mini kit (Qiagen GmbH, Hidden, Germany) was used for DNA extraction from approximately 200 mg stool samples according to the manufacturer instructions. Extracted DNAs were quantified by Qubit 2.0 Fluorimeter (Life Technologies, Carlsbad, CA, United States) using the Qubit dsDNA BR Assay Kit and stored at $-20^{\circ} \mathrm{C}$.

\section{Library preparation}

DNA libraries were prepared from 20 ng of DNA per sample using lon AmpliSeq Library Kit 2.0 (Life Technologies, California, US) following the kit guidelines. Ion AmpliSeq Colon and Lung Cancer panel v2 (Life Technologies, California, United States) consisting of a primer pool for analyzing 504 mutational hotspots from 92 amplicons was used to amplify the stool DNA. The genes included in this panel are: $A K T 1$, ALK, KRAS, BRAF, CTNNB1, DDR2, ERBB2, EGFR, ERBB4, FBXW7, NRAS, PIK3CA, PTEN, SMAD4, STK11, FGFR1, FGFR2, FGFR3, MAP2K1, $M E T, N O T C H 1$ and TP53 that are commonly mutated in colorectal cancer.

Amplified libraries were purified by Agincourt AM Pure XP beads (Beckman Coulter Genomics, High Wycombe, UK). Qubit 2.0 Fluorimeter and the Qubit dsDNA HS assay kit were used to quantify the barcoded libraries.

\section{Sequencing}

Before sequencing, templates were prepared using the lon OneTouch ${ }^{\mathrm{TM}} 2$ system (Life Technologies, California, United States); this system performs emulsion PCR for template amplification and its further purification and enrichment. Eventually, sequencing was accomplished using the lon PGM ${ }^{\text {TM }}$ Sequencing Hi-Q View Kit V2 and the amplified and enriched libraries loaded on lon $316 \mathrm{~S}$ chips and sequenced on the Ion Personal Genome Machine System (PGM ${ }^{\mathrm{TM}}$; Life Technologies, California, United States).

\section{Data analysis}

To analyze data and to evaluate run performance, Torrent suite software version 5.2.2 (Life Technologies) was used. Integrative Genomics Viewer (IGV v 2.2; Broad Institute, Cambridge, MA, United State) used for visual inspection of the aligned reads. All variants identified were further filtered by quality score ( $>15)$ and mutant allele frequency $(>5 \%)$. We used SIFT and PROVEAN in-silico tools to identify all those variants which resulted in non-synonymous, stop codon or frameshift amino acid changes and those that are predicted to have deleterious protein product. Previously reported somatic mutations and novel mutations were checked in COSMIC and dbSNP databases. Variants that are not recorded in either of the databases are labeled as 'Novel'. Kaplan-Meier analysis was performed to estimate the survival curve of the patients with different rate of mutations.

\section{Conclusion}

Early-stage stool DNA mutation analysis from CRC stage I/II patients would be a suitable, non-invasive approach for population specific screening. Our result shows TP53 and NRAS are the most frequently mutated genes in stool DNA of CRC patients from central part of Iran. Also comparing CRC and control group mutations can help to improve our understanding of mutations involved in cancer development mutation spectrum in other genes included in the NGS targeted panel that we used for this study is relatively different comparing to the previously reported mutation spectrum using the same NGS panel on ethnically different Iranian population as well as Finish CRC patients and controls. Our present data would help in development of stool-based CRC screening tools as well as more precisely selection of those who may benefit from anti EGFR monoclonal antibody therapy.

\section{Declarations}

\section{DATA AVAILABILITY}

All data generated or analyzed during this study are included in this published article and its Supplementary Information file.

\section{Acknowledgments}


Research reported in this publication was supported by Elite Researcher Grant Committee under award number 971202 from the National Institute for Medical Research Development (NIMAD), Tehran, Iran provided to Rasoul Salehi. The study was funded by the Sigrid Juselius Foundation grant to SK.

\section{Author Contributions}

FS, VS and OY contributed to acquisition and analysis of data and drafting the manuscript, AK, PP contributed to the design and execution of the work, SK, and RS contributed to the concept development, manuscript revision, data interpretation and approval.

\section{Corresponding Author}

Correspondence to Rasoul Salehi.

\section{Additional Information}

\section{Publisher's note}

Springer Nature remains neutral with regard to jurisdictional claims in published maps and institutional affiliations.

\section{Ethics Declarations}

\section{Competing interests}

The authors declare no potential conflicts of interest.

\section{References}

1. Ferlay, J. et al. Cancer incidence and mortality worldwide: IARC CancerBase. GLOBOCAN 2012, v10, 11 (2012).

2. Morson, B. The evolution of colorectal carcinoma. Clinical radiology, 35, 425-431 (1984).

3. Winawer, S. et al. Colorectal cancer screening: Clinical guidelines and rationale (Gastroenterology (1997) 112 (594-642))., 112, 1060 (1997). Erratum

4. Martínez, M. E. et al. A pooled analysis of advanced colorectal neoplasia diagnoses after colonoscopic polypectomy., 136, 832-841 (2009).

5. van Heijningen, E. M. B. et al. Features of adenoma and colonoscopy associated with recurrent colorectal neoplasia based on a large community-based study., 144, 1410-1418 (2013).

6. Wilson, J. M. G., Jungner, G. \& Organization, W. H. Principles and practice of screening for disease(1968).

7. Howlader, N. et al. SEER cancer statistics review, 1975-2013.Bethesda, MD: National Cancer Institute19 (2016).

8. Kuipers, E. J., Rösch, T. \& Bretthauer, M. Colorectal cancer screening-optimizing current strategies and new directions. Nature reviews Clinical oncology, 10, 130 (2013).

9. Brenner, H. et al. Reduced risk of colorectal cancer up to 10 years after screening, surveillance, or diagnostic colonoscopy., 146, 709717 (2014).

10. Joseph, D. A. et al. Prevalence of colorectal cancer screening among adults-behavioral risk factor surveillance system, United States, 2010. MMWR Morb Mortal Wkly Rep, 61, 51-56 (2012).

11. In Health at a Glance, > (2012).

12. Stock, C., Ihle, P., Schubert, I. \& Brenner, H. Colonoscopy and fecal occult blood test use in Germany: results from a large insurancebased cohort. Endoscopy, 43, 771-781 (2011).

13. Adler, A. et al. Improving compliance to colorectal cancer screening using blood and stool based tests in patients refusing screening colonoscopy in Germany. BMC gastroenterology, 14, 183 (2014). 
14. Lee-Six, H. et al. The landscape of somatic mutation in normal colorectal epithelial cells. Nature, 574, 532-537 (2019).

15. Kanthan, R., Senger, J. L. \& Kanthan, S. C. Molecular events in primary and metastatic colorectal carcinoma: a review. Pathology research international 2012 (2012).

16. Davies, R. J., Miller, R. \& Coleman, N. Colorectal cancer screening: prospects for molecular stool analysis. Nature Reviews Cancer, 5, 199 (2005).

17. Ahlquist, D. A. et al. The stool DNA test is more accurate than the plasma septin 9 test in detecting colorectal neoplasia. Clinical Gastroenterology and Hepatology, 10, 272-277 (2012).

18. Dickinson, B. T., Kisiel, J., Ahlquist, D. A. \& Grady, W. M. Molecular markers for colorectal cancer screening., 64, 1485-1494 (2015).

19. Imperiale, T. F. et al. Multitarget stool DNA testing for colorectal-cancer screening. New England Journal of Medicine, 370, 1287-1297 (2014).

20. Pox, C. Colon cancer screening: which non-invasive filter tests? Dig. Dis, 29, 56-59 (2011).

21. Beg, S. et al. Molecular markers and pathway analysis of colorectal carcinoma in the Middle East., 121, 3799-3808 (2015).

22. Salehi, R., Fisher, C. A., Bignell, P. A., Eslami, G. \& Old, J. M. Identification of Three Novel Mutations [- 41 (A > C), codon 24 (-G), and IVS-I-109 (- T)], in a Study of $\beta$-Thalassemia Alleles in the Isfahan Region of Iran., 34, 115-120 (2010).

23. Sarhadi, V. et al. Gut microbiota and host gene mutations in colorectal cancer patients and controls of Iranian and Finnish origin. Anticancer research, 40, 1325-1334 (2020).

24. Youssef, O. et al. Gene mutations in stool from gastric and colorectal neoplasia patients by next-generation sequencing. World journal of gastroenterology, 23, 8291 (2017).

25. Armengol, G. et al. Driver gene mutations in stools of colorectal carcinoma patients detected by targeted next-generation sequencing. The Journal of Molecular Diagnostics, 18, 471-479 (2016).

26. Kleist, B. et al. Comparison of neuroendocrine differentiation and KRAS/NRAS/BRAF/PIK3CA/TP53 mutation status in primary and metastatic colorectal cancer. International journal of clinical and experimental pathology, 7, 5927 (2014).

27. Cercek, A. et al. Clinical features and outcomes of patients with colorectal cancers harboring NRAS mutations. Clin. Cancer Res, 23, 4753-4760 (2017).

28. Tameshkel, F. S. et al. Mutation analysis of KRAS and BRAF genes in metastatic colorectal cancer: a first large scale study from Iran. Asian Pacific Journal of Cancer Prevention, 17, 603-608 (2016).

29. Payandeh, M., Sadeghi, M., Sadeghi, E. \& Gholami, F. Analysis of KRAS, BRAF and NRAS in patients with colorectal cancer: The first report of Western Iran. American Journal of Cancer Prevention, 3, 19-22 (2015).

30. Hamzehzadeh, L., Khadangi, F., Karimiani, E. G., Pasdar, A. \& Kerachian, M. A. Common KRAS and NRAS gene mutations in sporadic colorectal cancer in Northeastern Iranian patients. Current Problems in Cancer, 42, 572-581 (2018).

31. De Roock, W. et al. Effects of KRAS, BRAF, NRAS, and PIK3CA mutations on the efficacy of cetuximab plus chemotherapy in chemotherapy-refractory metastatic colorectal cancer: a retrospective consortium analysis. The lancet oncology, 11, 753-762 (2010).

32. Jouini, R. et al. KRAS and NRAS pyrosequencing screening in Tunisian colorectal cancer patients in 2015. Heliyon, 5, e01330 (2019).

33. Guo, F. et al. Mutation status and prognostic values of KRAS, NRAS, BRAF and PIK3CA in 353 Chinese colorectal cancer patients. Scientific reports, 8, 6076 (2018).

34. Peeters, M. et al. Massively parallel tumor multigene sequencing to evaluate response to panitumumab in a randomized phase III study of metastatic colorectal cancer. Clin. Cancer Res, 19, 1902-1912 (2013).

35. Dos Santos, W. et al. Mutation profiling of cancer drivers in Brazilian colorectal cancer. Scientific reports, 9, 1-13 (2019).

36. Lee, C. S. et al. Enhancing the landscape of colorectal cancer using targeted deep sequencing. Scientific reports, 11, 1-26 (2021).

37. Hsieh, J. C., Van Den Berg, D., Kang, H., Hsieh, C. L. \& Lieber, M. R. Large chromosome deletions, duplications, and gene conversion events accumulate with age in normal human colon crypts., 12, 269-279 (2013).

38. Blokzijl, F. et al. Tissue-specific mutation accumulation in human adult stem cells during life. Nature, 538, 260 (2016).

\section{Figures}




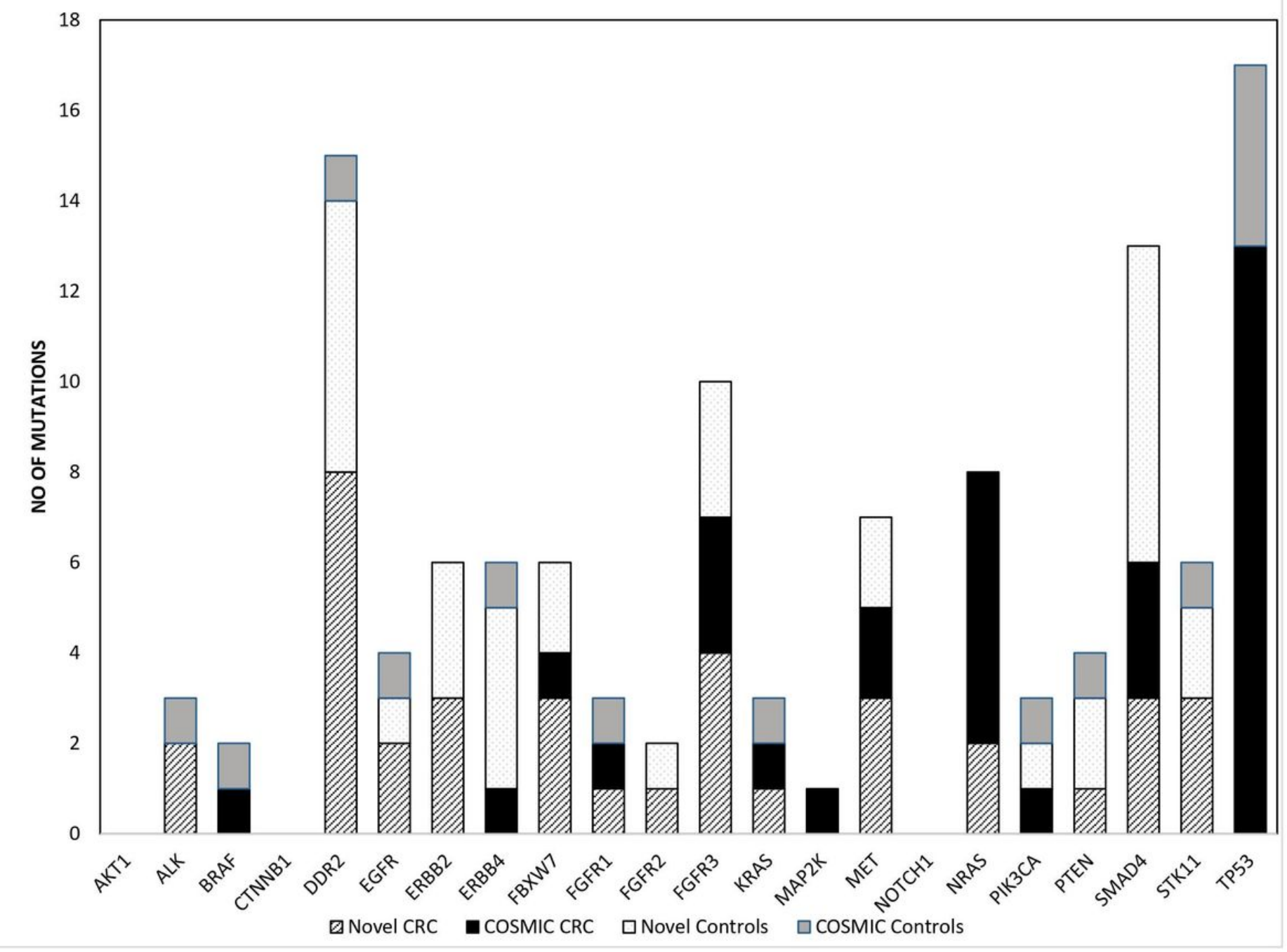

Figure 1

Genes with novel and COSMIC mutations in stool DNA of Iranian colorectal cancer patients and controls. 


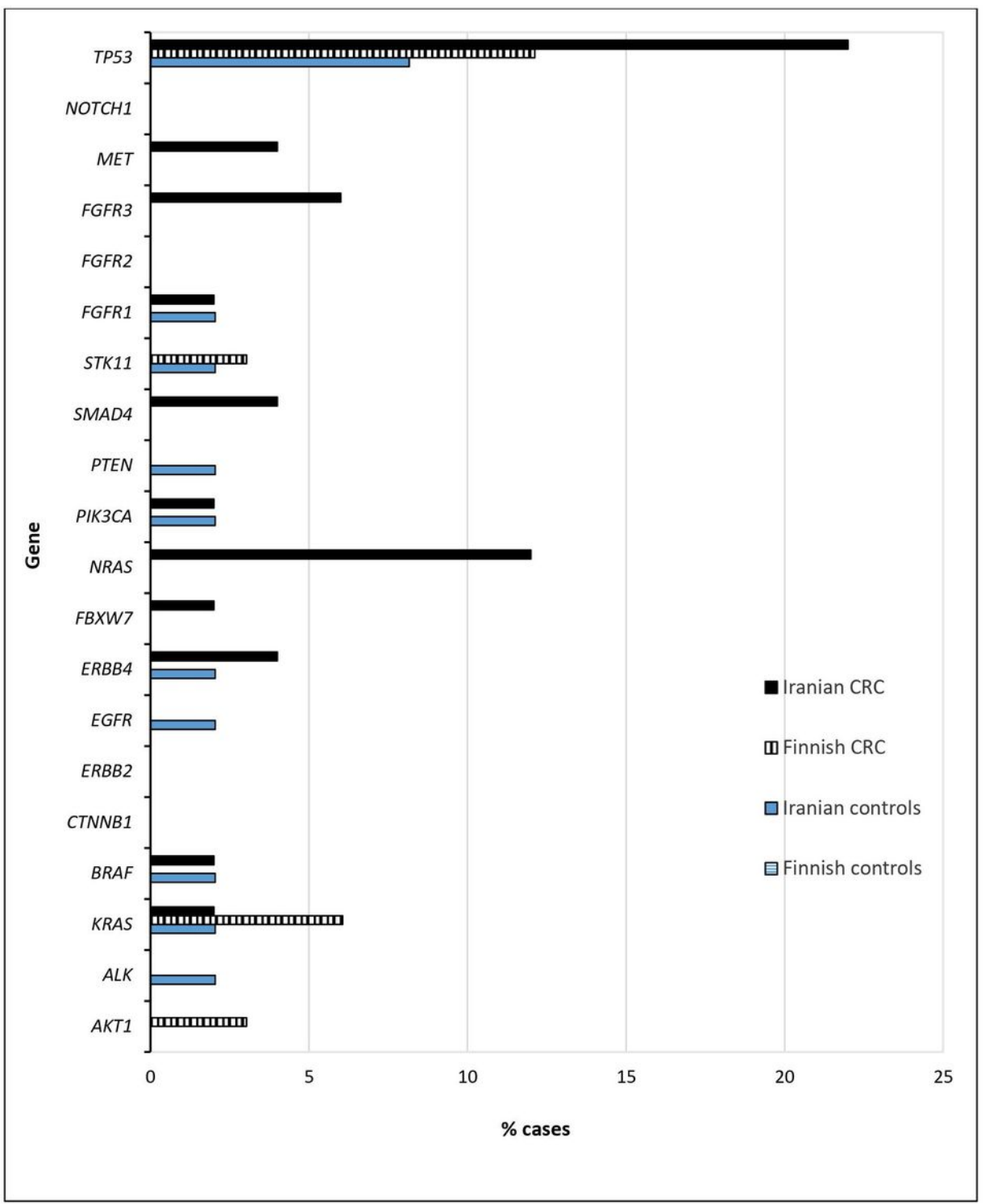

\section{Figure 2}

Comparison of hotspot mutations seen in stool DNA of colorectal cancer patients and controls of Iranian and Finnish origin

\section{Supplementary Files}

This is a list of supplementary files associated with this preprint. Click to download.

- SupplementaryDataset1.pdf 